\title{
Chronic thromboembolic pulmonary hypertension: do we need a new definition?
}

\author{
Marc de Perrot ${ }^{1}$ and Eckhard Mayer $^{2}$
}

Affiliations: 'Division of Thoracic Surgery, Toronto General Hospital, Toronto, ON, Canada. ${ }^{2}$ Dept of Thoracic Surgery, Kerckhoff Clinic, Bad Nauheim, Germany.

Correspondence: Marc de Perrot, Division of Thoracic Surgery, Toronto General Hospital, 200 Elizabeth Street, 9N-961, Toronto, ON, M5G 2C4, Canada. E-mail: Marc.DePerrotduhn.on.ca

@ERSpublications

Selection of patients for pulmonary endarterectomy in the absence of $\mathrm{PH}$ requires thorough evaluation http://ow.ly/CEO3M

Chronic thromboembolic pulmonary hypertension (CTEPH) is the end result of persistent obstruction of the pulmonary arteries following episodes of acute and/or recurrent pulmonary emboli [1]. Pulmonary hypertension probably stems from a smaller pulmonary arterial cross sectional area due to thrombus organisation and a redistribution of blood flow through the patent pulmonary arterial bed producing an additional microvasculopathy [2]. Consequently many patients develop pulmonary hypertension despite adequate anticoagulation [3]. Once pulmonary hypertension occurs, the prognosis is poor due to right heart failure with a survival similar to other forms of pre-capillary pulmonary hypertension [4]. Patients suffering from CTEPH typically present with progressive dyspnoea that worsens over a period of months to years [5]. Dyspnoea is related to hypoxaemia, dead space ventilation and right heart dysfunction, severely affecting the quality of life $[6,7]$.

Pulmonary endarterectomy is the treatment of choice for CTEPH. The surgery leads to major clinical improvement due to improved haemodynamic parameters and oxygenation, and a reduction in dead space ventilation $[7,8]$. The surgery is curative in a large proportion of patients with resolution of the pulmonary hypertension. Over the past 20 years, the technique of pulmonary endarterectomy under deep hypothermic circulatory arrest has been refined and can currently be performed with reproducible results across an increasing number of specialised centres. Expert centre series and a recent prospective multicentre registry demonstrated that pulmonary endarterectomy can be carried out safely in experienced centres with an overall operative mortality of $<5 \%[8,9]$. The risk of surgery depends of the severity of the disease and the mortality can be inferior to $1 \%$ in patients with limited vasculopathy and adequate right ventricular function $[10,11]$. Long-term results are favourable with regard to survival, exercise capacity and quality of life $[12,13]$.

In the era of successful surgical therapy, CTEPH, which was considered to be a rare entity, is being diagnosed more and more frequently. Several recent prospective studies have shown that the prevalence of CTEPH can be as high as 5\% after acute pulmonary embolism $[14,15]$. In addition, following an episode of acute pulmonary emboli a growing number of patients are being identified with persistent dyspnoea in the absence of pulmonary hypertension (mean pulmonary arterial pressure $<25 \mathrm{mmHg}$ at rest), affecting their exercise capacity and quality of life [16]. The symptoms of breathlessness in these patients can be related to excessive dead space ventilation, pulmonary hypertension on exercise and/or maladaptation of the right

Received: Sept 262014 | Accepted after revision: Oct 032014

Conflict of interest: Disclosures can be found alongside the online version of this article at erj.ersjournals.com 
ventricle to the decreased pulmonary artery compliance $[17,18]$. The prognosis and risk of progression to a more severe form of pulmonary hypertension, however, remains unclear.

Over the past few years, an increasing number of patients with symptomatic chronic thromboembolic disease (CTED) in the absence of pulmonary hypertension have undergone pulmonary endarterectomy in expert centres to improve their symptoms and quality of life. In this issue of the European Respiratory Journal, a study from the group in Cambridge systematically analysed the results of pulmonary endarterectomy in these patients for the first time [19]. The authors demonstrated the benefit of pulmonary endarterectomy and the significant impact on quality of life. Among 1019 patients undergoing pulmonary endarterectomy in their institution between 2000 and 2013, 42 underwent pulmonary endarterectomy for CTED in the absence of pulmonary hypertension at the time of their evaluation. Despite the fact that these patients were operated in one of the most experienced centres in the world, the rate of perioperative complications was still high $(40 \%)$ demonstrating the complexity of the surgical treatment concept. All patients were discharged from hospital alive and two died during follow-up. A total of 39 patients underwent a comprehensive review at 6 months after pulmonary endarterectomy and 31 had follow-up beyond 12 months. Their assessment showed a significant improvement in the New York Heart Association functional class and 6-min walking distance at 6 and 12 months follow-up. More importantly, using the Cambridge Pulmonary Hypertension Outcome Review questionnaire, the authors were able to demonstrate significant improvements in symptoms, activity levels and quality of life at 6 months after surgery that were sustained at 12 months.

Although the authors did not provide the denominator of patients with CTED assessed for pulmonary endarterectomy in their centre during the study period, these were probably a carefully selected group of patients as demonstrated by the small number of cases and relatively young age of the patients compared with series of patients with CTEPH undergoing pulmonary endarterectomy. This study does support the role of pulmonary endarterectomy in selected patients with CTED without pulmonary hypertension and highlights that, nowadays, the outcome of pulmonary endarterectomy should not only be looked at from a survival standpoint, but should also take into account quality of life and duration of benefit. Not offering pulmonary endarterectomy to patients with CTED due to the lack of pulmonary hypertension would potentially deprive many symptomatic patients of the chance to improve their quality of life. The individual expectation of pulmonary endarterectomy in patients with CTED is, however, different than in patients with CTEPH and careful selection of patients for surgery is important.

This evolution in the use of pulmonary endarterectomy leads to new questions pertaining to the definition of patients with symptomatic disease in the absence of pulmonary hypertension and their selection for surgery. For instance, should a new definition for CTEPH be determined that would encompass patients with CTED despite the lack of pulmonary hypertension at rest? Or should the terminology CTED be used for all patients with symptomatic disease with or without pulmonary hypertension rather than a more descriptive term such as chronic thromboembolic pulmonary arterial disease (CTEPAD)? The role of exercise in the selection of patients with symptomatic disease in the absence of pulmonary hypertension will also require further investigations.

At this stage, the selection of patients for pulmonary endarterectomy in the absence of pulmonary hypertension should be made after a thorough evaluation and individual discussion with the patients. The indication for surgery must be made based on patients' expectations and acceptance of risk. Pulmonary endarterectomy is a complex procedure that can be safely performed only in expert centres based on a high level of experience of an interdisciplinary team. This is even more important for the cohort of patients with CTED without pulmonary hypertension as their prognosis without surgical treatment is probably favourable.

\section{References}

1 Lang IM, Pesavento R, Bonderman D, et al. Risk factors and basic mechanisms of chronic thromboembolic pulmonary hypertension: a current understanding. Eur Respir J 2013; 41: 462-468.

2 Mercier O, Fadel E. Chronic thromboembolic pulmonary hypertension: animal models. Eur Respir J 2013; 41: 1200-1206.

3 de Perrot M, Fadel E, McRae K, et al. Evaluation of persistent pulmonary hypertension after acute pulmonary embolism. Chest 2007; 132: 780-785.

4 Riedel M, Stanek V, Widimsky J, et al. Longterm follow-up of patients with pulmonary thromboembolism. Late prognosis and evolution of hemodynamic and respiratory data. Chest 1982; 81: 151-158.

5 Pepke-Zaba J, Delcroix M, Lang I, et al. Chronic thromboembolic pulmonary hypertension (CTEPH): results from an international prospective registry. Circulation 2011; 124: 1973-1981.

6 Delcroix M, Vonk Noordegraaf A, Fadel E, et al. Vascular and right ventricular remodelling in chronic thromboembolic pulmonary hypertension. Eur Respir J 2013; 41: 224-232.

7 van der Plas MN, Reesink HJ, Roos CM, et al. Pulmonary endarterectomy improves dyspnea by the relief of dead space ventilation. Ann Thorac Surg 2010; 89: 347-352. 
8 Madani MM, Auger WR, Pretorius V, et al. Pulmonary endarterectomy: recent changes in a single institution's experience of more than 2,700 patients. Ann Thorac Surg 2012; 94: 97-103.

9 Mayer E, Jenkins D, Lindner J, et al. Surgical management and outcome of patients with chronic thromboembolic pulmonary hypertension: results from an international prospective registry. J Thorac Cardiovasc Surg 2011; 141: $702-710$.

10 Dartevelle P, Fadel E, Mussot S, et al. Chronic thromboembolic pulmonary hypertension. Eur Respir J 2004; 23: 637-648.

11 Jenkins DP, Madani M, Mayer E, et al. Surgical treatment of chronic thromboembolic pulmonary hypertension. Eur Respir J 2013; 41: 735-742.

12 Corsico AG, D’Armini AM, Cerveri I, et al. Long-term outcome after pulmonary endarterectomy. Am J Respir Crit Care Med 2008; 178: 419-424.

13 Yoshimi S, Tanabe N, Masuda M, et al. Survival and quality of life for patients with peripheral type chronic thromboembolic pulmonary hypertension. Circ J 2008; 72: 958-965.

14 Guérin L, Couturaud F, Parent F, et al. Prevalence of chronic thromboembolic pulmonary hypertension after acute pulmonary embolism. Thromb Haemost 2014; 112: 598-605.

15 Mehta S, Helmersen D, Provencher S, et al. Diagnostic evaluation and management of chronic thromboembolic pulmonary hypertension: a clinical practice guideline. Can Respir J 2010; 17: 301-334.

16 Klok FA, Tijmensen JE, Haeck ML, et al. Persistent dyspnea complaints at long-term follow-up after an episode of acute pulmonary embolism: results of a questionnaire. Eur J Intern Med 2008; 19: 625-629.

17 McCabe C, Preston SD, Gopalan D, et al. Cardiopulmonary exercise testing suggests a beneficial response to pulmonary endarterectomy in a patient with chronic thromboembolic obstruction and normal preoperative pulmonary hemodynamics. Pulm Circ 2014; 4: 137-141.

18 McCabe C, White PA, Hoole SP, et al. Right ventricular dysfunction in chronic thromboembolic obstruction of the pulmonary artery: a pressure-volume study using the conductance catheter. J Appl Physiol 2014; 116: 355-363.

19 Taboada D, Pepke-Zaba J, Jenkins DP, et al. Outcome of pulmonary endarterectomy in symptomatic chronic thromboembolic disease. Eur Respir J 2014; 44: 1635-1645. 